\title{
GAS CHROMATOGRAPHY: DEFINITIONS AND RETENTION PARAMETERS
}

\begin{abstract}
EADING workers in gas chromatography soon realized that published retention data would have much greater value if they were reported in a manner which made quantitative comparison possible, and in 1956, at the first committee meeting of the Gas Chromatography Discussion Group of the Institute of Petroleum Hydrocarbon Research Group, a sub-committee was formed to consider recommendations on presentation of data. Although differences between the views of this sub-committee and American workers were settled early in 1958, it was evident that international agreement was desirable, and approaches to the International Union of Pure and Applied Chemistry resulted in the formation of a Task Group to consider the matter. In addition to presentation of data, the Task Group also considered nomenclature, its terms of reference being to encourage the use of established conventions provided they were consistent with accepted theory, to select a single term where seversl existed, and to eliminate ambiguity of definition. The preliminary report of the Task Group was made available to the Discussion Group early in 1960 and has now been published in Pure and Applied Chemistry, the journal of the International Union.
\end{abstract}

At an informal symposium held at the University of Liverpool on October 21, where the Discussion Group was welcomed by Dr. G. Skirrow, the International Union of Pure and Applied Chemistry recommendations were discussed, and papers on alternative methods of presentation were read. The chair for the morning session was taken by $\mathrm{Mr}$. C. S. G. Phillips and for the afternoon session by $\mathrm{Mr}$. R. P. W. Scott.

After reviewing the historical background and acknowledging a valuable contribution to the work by the Groupement pour l'Avancement des Méthodes Spectrographiques, Dr. D. Ambrose, chairman of the International Union Task Group, discussed some of the points which had been considered in drawing up the recommendations summarized below.

The technique of gas chromatography is divided into gas-liquid and gas-solid, depending on whether separation is effected by partition or adsorption. Definitions are given for the following terms : sample injector, column (solid volume, liquid volume and interstitial volume), detector (differential and integ. ral), carrier gas, liquid phase, solid support and active solid. In gas-liquid chromatography the stationary phase comprises the liquid phase without solid support, and in gas-solid chromatography the stationary phase is the active solid. On the chromatogram, base line, peak, peak base, peak area, peak height, peak width and peak width at half-poek height are defined and illustrated. The Martin and James pressure gradient correction factor is designated $j$. Uncorrected and corrected (factor $j$ applied) retention volumes measured from the point of injection are respectively $V_{R}$ and $V^{0} R$. - The uncorrected retention volume for a non-absorbed gas is termed gas hold-up, $V_{M}$. The adjusted retention volume $V_{R^{-}}-V_{M}$ is designated $V_{R}^{\prime}$ and becomes net retention volume $V_{N}$ when the correction factor $j$ has been applied. These parameters are reported at the column temperature. Specific retention volume $V_{g}$ is the net retention volume per gram of liquid phase corrected to the volume at $0^{\circ} \mathrm{C}$.

Retention data should be published on an absolute basis or relative to a pure solute for which an absolute value is known at the operating temperature, and, wherever possible, the variation with temperature should be reported. Relative retention $r$ of two components is given by :

$$
r_{1,2}=\frac{V_{g_{1}}}{V_{g_{2}}}=\frac{V_{N_{1}}}{V_{N_{3}}}=\frac{V_{R_{1}}^{\prime}}{V_{R_{2}}^{\prime}} \neq \frac{V_{R_{1}}}{V_{R_{2}}}
$$

The theoretical plate velue $n$ of a column is used to express column performence (previously referred to as column efficiency), and peak resolution is given by twice the difference between retention (in volume, time or distance) divided by the sum of the peak widths in the corresponding units. Sample size, method of injection, and detector dead volume are factors which can affect the values obtained for retention parameters, column performance and resolution unless corrected for. The recommendations are preliminary and the Task Group is to exist for at least another two years; comment and suggestions will be welcomed by Dr. D. Ambrose (National Chemical Laboratory, Teddington, Middle. sex, England).

Mr. G. R. Primavesi (Distillers Co., Ltd.), expressing the views of an analyst on the presentation of data, maintained that whatever terms were used they should be relative as well as absolute, and that chart distance took pride of place over time or volume. The theoretical treatment of Martin and James was adequate, although the analyst had no need for the term $V^{0} R$. Retardation volume was preferred to net retention volume, and a plea was made that the expression for calculating $j$ be published in a form which could not be misinterpreted. Mr. Primavesi commented on the modes of presentation of specific retentions, and expressed the view that it was more convenient to have retention data measured at the column temperature.

Replying to these and other comments which hed been received, Dr. Ambrose stated that, although the relation of partition coefficient to retention data is included in the recommendations, it is not regarded as a retention parameter but merely as an examplo of one of a number of physical constants which can be determined by gas chromatography. The Task Group had taken as a fundamental experimental quantity the retention volume $V_{R}$, which is a volume of gas ; the fact that the actual measurement was made in time or distance was irrelevant. The term $V^{0} R$ was retained with its original definition on historical grounds but with the qualification that "this 
quantity is of limited use because it is influenced by the volumes of sample injector and detector as well as the interstitial volume of the column", because Martin and James made no mention of the significance of messurement from the air peak and, in fact, defined the ratio $V_{R_{1}} / V_{R_{3}}$ as relative retention. Some confusion had arisen over the use of the term $V_{g}$; it had been used to describe specific retention per gram at the column temperature and at $0^{\circ} \mathrm{C}$. There was very little to choose between the two interpretations, and the latter was chosen for recommendation because flow-meters are usually calibrated at $0^{\circ} \mathrm{C}$ and the plot of $V_{g}$ at $0^{\circ} \mathrm{C}$. against $1 / T$ is linear. Authors are advised to indicate the definition of $V_{g}$ used when reporting results.

Speaking as a teacher requiring a logically correct approach to theory, Prof. P. Chovin (Laboratoire Municipal, Paris, and chairman of Groupement pour l'Avancement des Méthodes Spectrographiques) preferred to develop it from an ideal column without external dead volume in which retention volume is a 'true' value. This concept does not affect adjusted, absolute and specific velues. However, in the case of column performarce where retention is measured from the point of injection, comparison of the 'true' and 'real' values gives valuable in. formation on the loss of performance resulting from parts of the apparatus external to the column. The difficulties associated with the measurement of effective injector and detector dead volumes limit the practical interest in 'true' retention parameters. Prof. Chovin also discussed a new parameter, net retention volume per mole of liquid phase, and its application to the determination of activity coefficients. If the activity coefficient of a solventsolute system at a given temperature was known, the value for the same solute with a different solvent could be determined very simply, and for this purpose a dual column assembly in one vapour jacket had been used.

In a paper by Mr. R. L. Martin (Standard Oil Co., of Indiana), read by Dr. A. Goldup, work was described which was interpreted as indicating adsorption effects at the liquid-gas interface. Such a phenomenon seriously affects the derivation of partition coefficients and other derived thermodynamic functions unless corrected for. In some circumstances the correction could amount to more than 80 per cent of the measured retention volume. With packed columns it had been found that the order of elution of hydrocarbon solutes varied with the amount of liquid phase, and the plot of retention volume per gram of column-packing versus liquid-phase percentage did not extrapolate to zero retention at zero liquid phase. With non-polar liquid phases the deviations were small and were attributable to adsorption on the support. With polar liquid phases the deviations were considerably larger and varied with the liquid phase. Using values for liquid surface area obtained. by subjecting the coated support to the gas chromatographic continuous-flow method for surface area of solids, retention volumes were accounted for as the sum of a contribution by solution in the liquid (partition coefficient $x$ volume of liquid) and a contribution by adsorption on the surface of the liquid (adsorption coefficient $\times$ surface area of liquid). The contribution by solution increased linearly with increase of liquid phase, but the contribution by adsorption decreased due to the decrease in liquid surface area as the percentage of liquid phase increased. Calculated 'adsorbabilities' for saturated hydrocarbons on polar liquid phases bore an inverse relation to the surface tension of the hydrocarbons. It was also noted that column performance was considerably greater with liquids on which adsorption occurred.

Mr. W. T. Swanton (British Petroleum Co.) ques. tioned the validity of some of the arguments, and indicated that effects due to the solid support, uncoated support in perticular, should be considered carefully. The relative values obtained for some of the 'adsorbabilities' were also queried; static experiments and application of the Gibbs adsorption equation might clarify the position. Further discussion on this interesting paper was limited by the absence of the author.

Dr. E. Kovets and Dr. J. F. Smith each presented mothods for reporting data which go a long way towards solving the difficulties of analysts, who so often find that their extensive collection of relative values is of limited application because it lacks a common denominator relating the different standards which have been used to cover liquid phases of diverse type and a variety of temperatures.

In the Retention Index Scheme proposed by Dr. Kovats (Laboratory for Organic Chemistry, Zurich) the retention of a solute is given as a value obtained by logarithmic interpolation between the values for two even-numbered $n$-alkanes. which, for convenience, are assigned values equivalent to one hundred times their carbon number. The good repeatability and reproducibility of the method were illustrated with the aid of values celculated from retention data publshed by other workers ; however, as with other forms of retention data, possible effects of solid support must be considered and care taken in standardizing on a reproducible support. Except for small molecules such as methanol and nitroethane, where the functional group dominates, the variation of retention index with column temperature is small and linear-about four units per ten degroes centigrade. From the results of a large number of determinations, the difference between the retention indexes of two close-boiling isomers is deduced as five times the difference between their boiling points $\left({ }^{\circ} \mathrm{C}\right.$.). Retention indexes increase by one hundred units on both polar and non-polar phases for the higher members of any homologous series with the insertion of $\mathrm{a}-\mathrm{CH}_{2}$ group. Differences in values obtained on polar and non-polar phases can be ascribed to solute type. For non-polar hydrocarbons the difference is zero. Dr. Kovats presented evidence that bonding types and functional groups in a molecule can each be assigned a difference value, which for a given polar liquid phase are additive.

With Dr. Smith's theoretical nonane or $R_{x g}$ method, which has been used extensively and proved invaluable in the laboratories of the Natural Rubber Producers' Research Association, all values are expressed relative to the retention time for $n$-nonane, which is obtained by extrapolation or interpolation from the logarithmic retention time versus carbon number plots with $n$-alkanes. A simple relation between the $R_{x g}$ value and the retention index was shown to exist. Statistical examination of the results obtained for various chemical classes on squalane and dinonyl phthalate at several temperatures showed that the repeatability for the whole calibration procedure and determination of relative value were at least as good as for methods involving the use of one or more standards within the chromatogram.

C. G. Scort 